\title{
Optimized generation of survivin-specific cytotoxic T lymphocytes against lung cancer
}

\author{
YING LI and JUANJUAN DING \\ Department of Respiratory Medicine, Henan Provincial People's Hospital, Zhengzhou, Henan 450003, P.R. China
}

Received January 5, 2014; Accepted September 4, 2014

DOI: $10.3892 / \mathrm{mmr} .2015 .3579$

\begin{abstract}
Cancer immunotherapy based on dendritic cells (DCs) that target survivin is a promising strategy with potential clinical applications. However, the translation of survivin-specific cytotoxic T-lymphocyte (CTL) immunotherapy into the clinical setting has numerous challenges, including the low efficiency of the treatment. The present study aimed to improve the efficiency of the treatment, and found that treatment with interleukin 4 (IL-4)/granulocyte macrophage colony-stimulating factor (GM-CSF) and a combination of proinflammatory cytokines significantly increased the antigen-presenting and -capture abilities of DCs that expressed exogenous survivin. Furthermore, lipopolysaccharide (LPS) stimulation enhanced the DC response to subsequent T-cell signals and the extent of T-cell activation. In addition, the efficiency of survivin-specific CTLs was examined, and high cytotoxicity against survivin-expressing A549 lung cancer cells was observed. However, the cytotoxicity of CTLs was significantly reduced in A549 cells with silenced survivin expression. The present study provides a novel method to optimize the generation of survivin-specific CTLs against lung cancer cells, which may advance the translation of survivin-specific CTL immunotherapy into clinical use for the treatment of cancer.
\end{abstract}

\section{Introduction}

A number of promising strategies to treat cancer have been developed over the past few decades (1-4). Among these approaches, immunotherapy provides safe, efficient and long-lasting effects (3). Cancer immunotherapy uses the immune system of the patient to attack malignant tumor cells (5). To achieve this, three approaches have been developed: Cancer vaccine-based immunization of the patient,

Correspondence to: Dr Ying Li, Department of Respiratory Medicine, Henan Provincial People's Hospital, No. 7 Weiwu Road, Zhengzhou, Henan 450003, P.R. China

E-mail: liying.ying@hotmail.com

Key words: survivin, dendritic cells, cytotoxic T lymphocytes, lung cancer therapeutic antibody-dependent recruitment of the patient's immune system and cell-based activating immune cells $(3,5)$. Recently, cell-based immunotherapy, for example, using dendritic cell (DC)-induced tumor antigen-specific cytotoxic $\mathrm{T}$ lymphocytes (CTLs), has become an attractive approach in cancer therapy (6). The success of DC-based immunotherapy depends on the identification of a tumor-specific antigen.

Survivin, a tumor-specific antigen, is highly expressed in the majority of malignant cancer types, including lung, pancreas, colon and prostate cancer. Survivin is crucial for the survival and proliferation of tumor cells; however, its expression is limited in normal tissues (7). Overexpression of survivin in tumors is associated with poor prognosis and is correlated with resistance to several types of anticancer therapy (8-10). Due to these findings, survivin has become an attractive candidate for tumor-specific therapy, and approaches that target survivin have been developed in recent years. For example, adenovirus-mediated transfer of small interfering (si)RNA against survivin has been demonstrated to induce tumor cell apoptosis and reduce tumor growth (11). The survivin peptide-pulse approach and the transduction of survivin into DCs have also been investigated for DC-based immunotherapy in human urological cancer cells $(12,13)$. Although these approaches have achieved marked progress in survivin-specific immunotherapy in certain types of cancer cells, optimization of these approaches is required to achieve efficient therapy. Furthermore, the efficiency of DC-based immunotherapy requires examination in other types of cancer cells, such as lung cancer cells, to expand the application of this treatment.

In the present study, an adenovirus system was used to overexpress the survivin gene in DCs derived from peripheral blood mononuclear cells (PBMCs). Survivin-overexpressing DCs were treated with interleukin 4 (IL-4)/granulocyte macrophage colony-stimulating factor (GM-CSF) and a combination of proinflammatory cytokines. Subsequent to treatment, the survivin-specific CTLs that were induced by survivin-overexpressing DCs were examined.

\section{Materials and methods}

Reagents and antibodies. Reagents were obtained from the following sources: RPMI-1640 medium, Dulbecco's modified Eagle's medium (DMEM) and fetal bovine serum (FBS) were HyClone ${ }^{\mathrm{TM}}$ products (GE Healthcare, Logan, UT, USA); 
GM-CSF, IL-4 and ELISA kits for tumor necrosis factor (TNF)- $\alpha$ (cat. no. BMS223/4) and IL-12 (cat. no. BMS616) were bought from eBioscience, Inc. (San Diego, CA, USA); lipopolysaccharide (LPS) and fluorescein isothiocyanate (FITC)-dextran (cat. no. 46945-100MG-F) were obtained from Sigma-Aldrich (St. Louis, MO, USA); and control siRNA and survivin siRNA were provided by Life Technologies (Carlsbad, CA, USA). Anti-CD14 (ab45870), anti-CD16 (ab94773), anti-CD86 (ab53004), anti-CD80 (ab64116), and major histocompatibility complex (MHC) I (ab52992) and MHC II (ab116378) antibodies were purchased from Abcam (Cambridge, MA, USA); and survivin (product no. 2803) and actin (product no. 8456) antibodies were obtained from Cell Signaling Technology, Inc. (Danvers, MA, USA).

Cell culture. All DCs and DC-derived cells were cultured in RPMI-1640 with 10\% FBS. A549, 293, MDA-MB-231, HCT116 and H460 cells were purchased from the American Type Culture Collection (Manassas, VA, USA) and cultured in DMEM with $10 \%$ FBS. All cells were maintained in an incubator with $5 \% \mathrm{CO}_{2}$ at $37^{\circ} \mathrm{C}$.

Generation of DCs. DCs were obtained from the fresh heparinized peripheral blood of healthy donors, as previously described $(12,14)$. Briefly, PBMCs were isolated from the blood of the healthy volunteers by density gradient centrifugation with Ficoll-Paque (GE Healthcare), and were then cultured in RPMI-1640 complete medium supplied with $20 \mathrm{ng} / \mathrm{ml} \mathrm{GM-CSF}$ and $10 \mathrm{ng} / \mathrm{ml} \mathrm{IL-4,} \mathrm{for} \mathrm{seven} \mathrm{days.}$ Subsequently, fluorescence-activated cell sorting (FACS) was employed to examine the expression of CD14 and CD16 in these cells. For combined proinflammatory cytokine stimulation, the DCs were incubated with GM-CSF and IL-4 for two days, followed by treatment with $10 \mathrm{ng} / \mathrm{ml} \mathrm{TNF}-\alpha$ (cat. no. 210-TA-005; R\&D Systems, Minneapolis, MI, USA) for another two days. The DCs were then incubated with $100 \mathrm{ng} / \mathrm{ml} \mathrm{LPS} \mathrm{for} 48 \mathrm{~h}$.

Overexpression and knockdown of the survivin gene. The recombinant adenoviral vector pAdTrack harboring survivin, which was packaged and purchased from Yrbio, Inc. (Changsha, China), was transfected into the 293 cells (packaging cells) to generate a virus. DCs were infected with control or survivin-loading virus at multiplicity of infection (MOI) of $10,25,50$ or 100 . Subsequently, the cells were centrifuged at $1,000 \mathrm{x} \mathrm{g}$ at $37^{\circ} \mathrm{C}$ for $1 \mathrm{~h}$. Survivin expression levels were examined after $48 \mathrm{~h}$ using western blot analysis. For survivin knockdown, A549 cells were transfected with control siRNA or survivin-specific siRNA using Lipofectamine ${ }^{\circledR} 2000$ (Invitrogen Life Technologies, Carlsbad, CA, USA). The effects of survivin knockdown were verified by western blotting.

Western blot analysis. Total cell lysates were extracted using lysis buffer containing $2 \%$ sodium dodecyl sulfate, $10 \%$ glycerol, $10 \mathrm{~mm}$ Tris pH 6.8 and $100 \mathrm{~mm}$ dithiothreitol. The samples were boiled for $10 \mathrm{~min}$ and then subjected to immunoblotting as described previously (15).

Generation of CD40L stable line. To establish stable lines expressing CD40L in 293 cells, human CD40L gene was cloned into a p3xFLAG-CMV ${ }^{\mathrm{TM}}-10$ vector (cat no. E4401; Sigma-Aldrich) using the polymerase chain reaction through the BamHI restriction enzyme site. PCR reactions were performed using Extensor Long PCR ReddyMix Master mix with buffer 1 (Thermo Fisher Scientific, Waltham, MA, USA; cat. no. AB-0794/B) according to the manufacturer's instructions. For each $25 \mu \mathrm{l}$ reaction, the CD40L gene fragment was obtained by mixing the following reagents: 10X Extensor buffer $1(1.25 \mu \mathrm{l})$; dNTP mix, $2 \mathrm{mM}$ each $(3.125 \mu \mathrm{l})$; forward primer 5'CGCGGATCCATACAACCAAACT3'(0.5 $\mu 1$,final:200nM), reverse primer 5' TGAGTTTGAGACTCCTAGGCGC 3' (0.5 $\mu$ l, final: $200 \mathrm{nM})$, extensor PCR enzyme mix $(0.125 \mu \mathrm{l})$. The primers were synthesized by Sangon Biotech Co., Ltd. (Shanghai, China). cDNA was prepared using TRIzol ${ }^{\circledR}$ reagent (Invitrogen Life Technologies) according to the manufacturer's instructions template $(5 \mu \mathrm{l}, 100 \mathrm{ng}$ ), water (to $25 \mu \mathrm{l}$ ). PCR cycling conditions were as follows: Initial denaturation at $94^{\circ} \mathrm{C}$ for $2 \mathrm{~min}$, followed by 25 cycles of denaturation at $94^{\circ} \mathrm{C}$ for $10 \mathrm{sec}$, annealing at $60^{\circ} \mathrm{C}$ for $30 \mathrm{sec}$ and extension at $68^{\circ} \mathrm{C}$ for $2 \mathrm{~min}$, and a final extension at $68^{\circ} \mathrm{C}$ for $7 \mathrm{~min}$.

The 293 cells were cultured to $80 \%$ confluence in $60-\mathrm{mm}$ dishes prior to transfection with $3 \mu \mathrm{g}$ CD40L plasmid or empty vector for $48 \mathrm{~h}$ using Lipofectamine ${ }^{\circledR} 2000$ (Invitrogen Life Technologies). Subsequently, $800 \mu \mathrm{g} / \mathrm{ml}$ Geneticin (G418, cat no. A1720-1G; Sigma-Aldrich) was added to the culture medium to select clones expressing the neo gene in CD40L-plasmid- or empty-vector-transfected 293 cells for two weeks. The medium was replaced and fresh $\mathrm{G} 418$ was added every two days until the cells reached $80 \%$ confluence. The best 2-3 clones (as determined by high expression of CD40L) were transferred to T-75 culture flasks (Corning Inc., Corning, NY, USA) for scale-up production. The stable cell lines were used for the LPS stimulation experiments, as described for the DCs.

ELISA. The supernatants of the DC cultures under the different conditions were collected. TNF- $\alpha$ and IL-12 concentrations were measured with the respective specific ELISA kits according to standard procedures.

Generation of CTLs and CTL assay. PBMCs from fresh heparinized peripheral blood were separated by density gradient centrifugation with Ficoll-Paque. The cells were cultured in RPMI-1640 medium for $2 \mathrm{~h}$. The adherent fraction of PBMCs was used for the generation of DCs as described above. The nonadherent fraction was collected for CTL generation. DCs with or without survivin expression were seeded in 24-well plates $\left(2 \times 10^{5}\right.$ cells/well) and incubated with the corresponding nonadherent PBMCs at a ratio of 1:10 for seven days. These cells were collected and restimulated with survivin-expressing or control DCs. Cytotoxic activity to A549 cells was then analyzed using a CytoTox 96 Non-Radioactive Cytotoxicity assay kit (Part\# TB163, Promega Corporation, Madison, WI, USA) according to the manufacturer's instructions.

Flow cytometry and evaluation of antigen capture ability. The cells were analyzed with the corresponding antibodies using a BD FACSCalibur ${ }^{\mathrm{TM}}$ (BD Biosciences, San Jose, CA, USA) flow cytometer according to standard procedures. The 
A

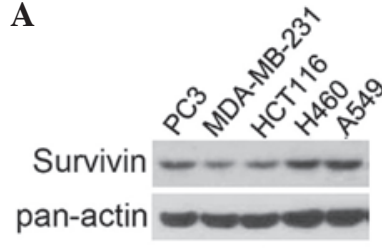

B

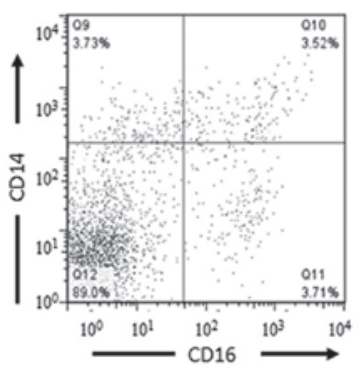

C

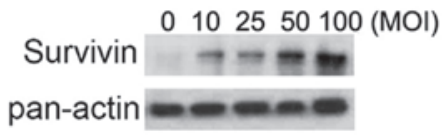

Figure 1. Generation of DCs with survivin overexpression. (A) Survivin protein expression levels in various cancer cell lines. (B) The percentages of CD14 and CD16-expressing cells in cultures were detected by fluorescence-activated cell sorting subsequent to culture of peripheral blood mononuclear cells for seven days in vitro: CD14/CD16 double-negative, 89\%; CD14/CD16 double-positive, 3.52\%; CD14-positive and CD16-negative, 3.73\%; CD14-negative and CD16-positive, 3.71\%. (C) Survivin protein levels in DCs infected by different doses of pAd-survivin adenovirus. DCs, dendritic cells; MOI, multiplicity of infection.

antigen-capture ability was determined by the FITC-dextran uptake as previously described (16). The cells were incubated with $0.5 \mathrm{mg} / \mathrm{ml}$ FITC-dextran for $2 \mathrm{~h}$ at $37^{\circ} \mathrm{C}$. Subsequently, after washing with PBS three times, the cells were analyzed by FACS. The FITC-dextran uptake was quantified as the mean fluorescence intensity.

Data analysis and presentation. All experiments were repeated at least three times, independently. The data were analyzed using GraphPad Prism 5 software (GraphPad Software, Inc., La Jolla, CA, USA) and are presented as the mean $\pm \mathrm{SD}$. $\mathrm{P}<0.05$ was considered to indicate a statistically significant difference.

\section{Results}

Generation of survivin-overexpressing DCs. To confirm the expression of survivin in the different cancer cell lines, survivin protein levels were examined. As shown in Fig. 1A, survivin was highly expressed in all cancer cell lines, particularly in the H460 and A549 lung cancer cells (Fig. 1A). This result suggests that survivin is a potential target for immunotherapy in this type of cancer. To develop DC-based immunotherapy, fresh PBMCs were isolated from healthy human donors and induced to differentiate into DCs. FACS was employed to examine the differentiation of PBMCs into DCs. The results revealed that the majority of cells (89\%) lost the ability to express CD14 and CD16 (Fig. 1B), suggesting these cells were differentiated (17). To obtain survivin-expressing DCs, the generated DCs were infected by an adenoviral vector loaded with survivin. Survivin expression in the DCs was verified by western blotting, which demonstrated that survivin expression levels were increased when DCs were infected at a higher MOI (Fig. 1C). An increased MOI elevated the survivin expression
A

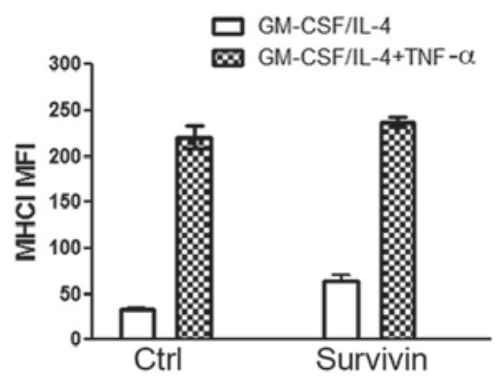

B

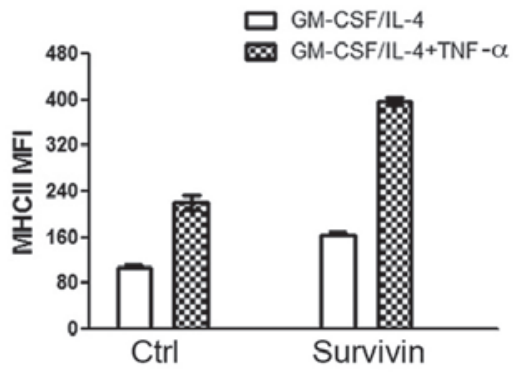

C

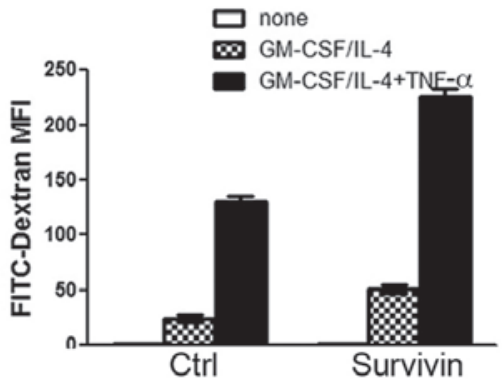

Figure 2. GM-CSF/IL-4/TNF- $\alpha$ enhanced the antigen-presenting and antigen-capture ability of DCs. (A and B) Expression levels of MHC (A) class I and (B) class II in control (Ctrl) or survivin-overexpressing (Survivin) DCs was detected subsequent to treatment with $20 \mathrm{ng} / \mathrm{ml} \mathrm{GM-CSF}$ and $10 \mathrm{ng} / \mathrm{ml}$ IL-4 for seven days, followed by the addition of $10 \mathrm{ng} / \mathrm{ml} \mathrm{TNF}-\alpha$ for another two days. (C) DCs (same as A and B) were incubated with FITC-dextran $(0.5 \mathrm{mg} / \mathrm{ml})$ for $2 \mathrm{~h}$ at $37^{\circ} \mathrm{C}$, FITC-dextran uptake was quantified as the MFI. GM-CSF, granulocyte macrophage colony-stimulating factor; IL, interleukin; TNF, tumor necrosis factor; DCs, dendritic cells; MHC, major histocompatibility complex; FITC, fluorescein isothiocyanate; MFI, mean fluorescence intensity.

levels in the DCs, and also decreased cell survival (data not shown). To balance the expression levels and toxic effects of survivin treatment, 50 MOI was selected for subsequent experiments. In summary, these results confirm the expression of survivin in different cancer cell lines and the generation of survivin-overexpressing DCs.

Sequential treatment with IL-4/GM-CSF and a combination of proinflammatory cytokines increases the antigen-presenting and antigen-capture ability of DCs. To achieve maximized efficiency of DC-based survivin-specific immunotherapy, the generation of survivin-overexpressing DCs requires optimization. To achieve this optimization, the antigen-presenting and -capture abilities of induced DCs first require improvement. GM-CSF and IL-4 were added to PBMC cultures, and the cultures were incubated for two days. This was followed by incubation for another two days subsequent to the administration of a combination 


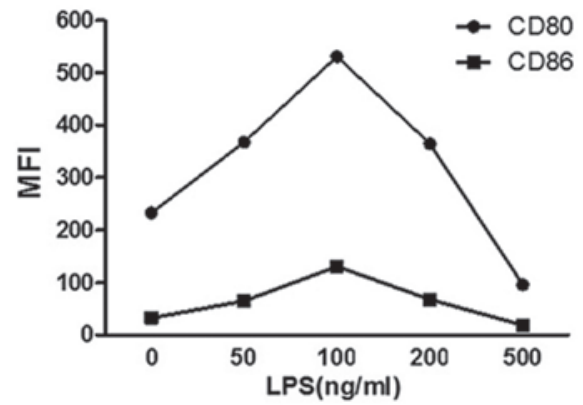

Figure 3. Expression levels of activation markers CD80 and CD86 were increased in DCs following LPS stimulation. The expression levels of CD80 and CD86 in DCs were examined by fluorescence-activated cell sorting. DCs, dendritic cells; LPS, lipopolysaccharide; MFI, mean fluorescence intensity.

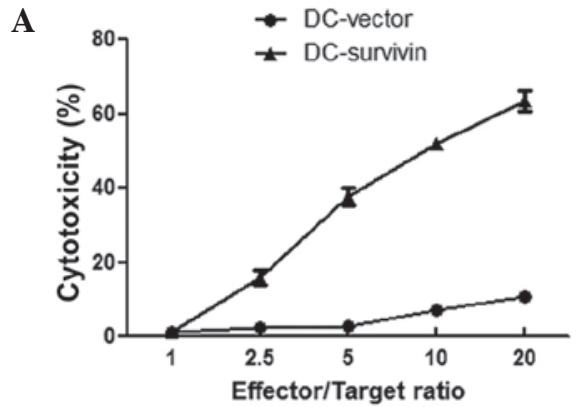

$\mathbf{B}$

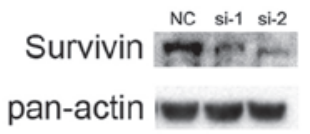

C

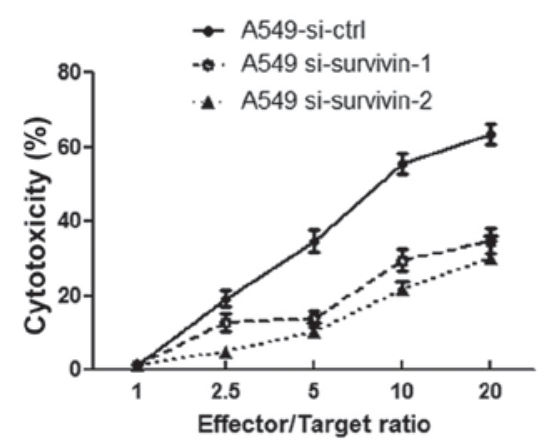

Figure 5. Survivin-specific CTL-induced DCs exhibited enhanced cytotoxicity against A549 cells. (A) CTL cytotoxicity to A549 cells induced by DCs with (DC-survivin) or without (DC-vector) survivin transfection. (B) Survivin protein levels in A549 cells following transfection of control siRNA (NC) or survivin-specific siRNA (si-1, si-2). (C) Survivin-specific cytotoxic effects of CTLs to A549 cells transfected with control siRNA (A549-si-ctrl) or survivin-specific siRNAs (A549-si-survivin-1 or A549-si-survivin-2). CTL, cytotoxic T lymphocyte; DCs, dendritic cells; siRNA, small interfering RNA.

Whether the DC antigen capture ability was affected by the treatments was then analyzed. The FACS results revealed that the DCs treated with both GM-CSF/IL-4 and proinflammatory cytokine captured more FITC-dextran than DCs treated with GM-CSF/IL-4 alone (Fig. 2C). Notably, DCs with survivin expression exhibited a more marked ability to capture FITC-dextran subsequent to treatment than the control DCs (Fig. 2C). In conclusion, treatment with GM-CSF/IL-4 and proinflammatory cytokines significantly increased the antigen-presenting ability of the DCs. These treatments also enhanced the antigen-capture ability of the DCs, particularly for DCs with survivin overexpression.

LPS stimulation increases cytokine secretion by DCs and sensitizes DCs for the subsequent activation of T cells. After the DC antigen-presenting and antigen-capture abilities had been increased, an approach that sensitizes DCs to signals from $\mathrm{T}$ cells was required. In mouse DCs, stimulation with LPS sensitizes the cells to signals from T cells (18). Whether LPS also enhances the function of human DCs was thus investigated in the present study. To examine this hypoth-

esis, LPS at different doses $(0,50,100,200$ and $500 \mathrm{ng} / \mathrm{ml})$ survivin overexpression was enhanced by GM-CSF/IL-4 treatment following proinflammatory cytokine stimulation.

of proinflammatory cytokines. The data revealed that this strategy markedly increased the expression levels of MHC I and MHC II in DCs with and without survivin overexpression (Fig. 2A and B). However, the expression levels of MHC II were increased to a greater extent in the survivin-overexpressing DCs as compared with the control DCs. These data indicate that the antigen-presenting ability of DCs with 
was added to DCs. The expression levels of CD80 and CD86, which are required for the activation and survival of T cells (19), were measured in DCs with or without LPS treatment. The expression levels of CD80 and CD86 were increased following LPS stimulation, and reached a peak with $100 \mathrm{ng} / \mathrm{ml}$ LPS stimulation (Fig. 3). In addition, the expression levels of CD80 and CD86 were higher in survivin-expressing DCs than in control DCs following LPS stimulation (Fig. 4A). This result suggests that survivin-overexpressing DCs exhibit a marked ability to activate T cells. Survivin expression in DCs also increased the basal levels of the TNF- $\alpha$ and IL-12 secretion factors (Fig. 4B). To analyze how survivin-overexpressing DCs respond to T cell signals following pretreatment with LPS, DCs were treated with LPS for $24 \mathrm{~h}$ and then co-cultured with control 293 cells or CD40L-expressing 293 cells in the presence of IL-4. The data revealed that the secretion of TNF- $\alpha$ and IL-12 was markedly increased in LPS-primed cells when the cells were co-cultured with CD40L-expressing 293 cells, compared with when the cells were co-cultured with control 293 cells (Fig. 4C). Collectively, the data suggest that LPS treatment primed survivin-overexpressing DCs, and promoted the secretion of the TNF- $\alpha$ and IL-12 cytokines in these DCs. Through this treatment, DCs became more sensitive to activated T-cell signals.

Survivin-specific CTLs are induced against lung cancer cells by DCs with survivin overexpression. Subsequent to optimization of the functions of survivin-overexpressing DCs, the survivin-specific cytotoxicity against A549 lung cancer cells induced by these treated DCs was further examined. The DC cytotoxicity to A549 cells was measured using the CytoTox 96 Non-Radioactive Cytotoxicity assay kit. Various ratios of effector/target (CTL/A549) cells, as indicated in Fig. 5, were applied to analyze the CTL cytotoxicity to A549 cells. As compared with the control DCs, survivin-overexpressing DCs induced a markedly higher CTL cytotoxicity to A549 cells (Fig. 5A). To confirm the specificity of survivin-overexpressing DC-induced CTLs, survivin knockdown was conducted using two specific siRNAs that target the survivin gene in A549 cells (Fig. 5B). The results revealed that the cytotoxic effects of CTLs to A549 cells with survivin-knockdown were clearly reduced, as compared with the effects on control A549 cells (Fig. 5C), which demonstrates that the CTLs were survivin-specific. In conclusion, these data revealed that optimized DCs induced markedly more effective survivin-specific CTLs against A549 lung cancer cells.

\section{Discussion}

DC-based immunotherapy is an effective approach in the induction of anticancer immunity (20). Although DC-based immunotherapy has been investigated for over 10 years, the fundamental problems concerning optimization of this approach have not been solved (21). In order to improve the generation of DCs for immunotherapy, particularstrategies have been developed, including the generation of DCs from different cell types (22), the culture of cells with monocyte-conditioned medium (23), or the incubation of DCs with TNF- $\alpha$ or LPS to stimulate maturation $(24,25)$. In the present study, DC treatment with a combination of factors was employed. The cells were first treated with IL-4/GM-CSF, followed by incubation with a combination of proinflammatory cytokines during DC maturation. With this treatment, DCs exhibited increased antigen-presenting and antigen-capture abilities (Fig. 2). LPS was then used to sensitize the DCs and enhance the response to $\mathrm{T}$ cells (Figs. 3 and 4). Following these optimization treatments, DCs induced high T-lymphocyte toxicity against A549 lung cancer cells (Fig. 5). Although the present study provides a novel method for optimizing the generation of DC-induced CTLs against lung cancer cells ex vivo, the in vivo efficiency requires analysis in future studies.

The success of cancer immunotherapy also depends on the tumor-specific antigen. Survivin, an inhibitor of the apoptosis protein caspase, is overexpressed in various types of cancer cells, including lung, pancreas, colon and prostate cancer cells (7), a finding consistent with the result from the present study, which revealed survivin to be highly expressed in the corresponding cancer cell lines (Fig. 1A). Survivin is involved in the inhibition of apoptosis and the regulation of mitosis in cancer cells (25). Furthermore, the presence of high survivin expression levels in cancer cells has been clearly associated with a poor prognosis and resistance to anticancer agents, radiotherapy and antiandrogen therapy in prostate cancer cells (8-10). These studies suggest that survivin is an attractive target for cancer immunotherapy. Therefore, several strategies that target survivin in cancer cells have been developed. One strategy is to employ siRNA that targets survivin (11). Other studies have used survivin-peptide pulsed- or survivin-transduced DC-based immunotherapy $(12,13)$. However, the specificity of these strategies in targeting survivin-expressing cancer cells has not been examined. In the present study, the specificity of survivin-overexpressing DC-induced CTLs in lung cancer cells was analyzed. The results revealed that the CTLs specifically targeted the A549 lung cancer cells that expressed survivin but not those cells with survivin-knockdown (Fig. 5B and C). The results clearly indicate that the CTLs induced by survivin-overexpressing DCs acted specifically against survivin-expressing cancer cells, which suggests a potential targeting therapy for cancers with survivin expression.

In conclusion, in the present study, a generation of DCs transduced with survivin was optimized and the specificity of survivin-specific CTLs induced by the survivin-overexpressing DCs against lung cancer cells was verified. Therefore, this study provides an optimization strategy for survivin-positive malignancies and promotes the potential application of survivin-specific CLTs in clinical trials.

\section{Acknowledgements}

The authors would like to thank all other members in the laboratory for technical support and helpful analysis.

\section{References}

1. Lewis LD: Cancer therapeutics revisited; novel drugs targeting cell signalling pathways, genome wide association studies and other trials and tribulations. Br J Clin Pharmacol 76: 317-319, 2013. 
2. Sliwkowski MX and Mellman I: Antibody therapeutics in cancer Science 341: 1192-1198, 2013.

3. Snook AE and Waldman SA: Advances in cancer immunotherapy. Discov Med 15: 120-125, 2013.

4. Workman P, Al-Lazikani B and Clarke PA: Genome-based cancer therapeutics: targets, kinase drug resistance and future strategies for precision oncology. Curr Opin Pharmacol 13: 486-496, 2013

5. Waldmann TA: Immunotherapy: past, present and future. Nat Med 9: 269-277, 2003.

6. Bonaccorsi I, Pezzino G, Morandi B and Ferlazzo G: Novel perspectives on dendritic cell-based immunotherapy of cancer. Immunol Lett 155: 6-10, 2013.

7. Ambrosini G, Adida C and Altieri DC: A novel anti-apoptosis gene, survivin, expressed in cancer and lymphoma. Nat Med 3: 917-921, 1997.

8. Rodel F, Hoffmann J, Distel L, et al: Survivin as a radioresistance factor, and prognostic and therapeutic target for radiotherapy in rectal cancer. Cancer Res 65: 4881-4887, 2005

9. Su L, Wang Y, Xiao M, Lin Y and Yu L: Up-regulation of survivin in oral squamous cell carcinoma correlates with poor prognosis and chemoresistance. Oral Surg Oral Med Oral Patho Oral Radiol Endod 110: 484-491, 2010.

10. Zhang M, Latham DE, Delaney MA and Chakravarti A: Survivin mediates resistance to antiandrogen therapy in prostate cancer. Oncogene 24: 2474-2482, 2005.

11. Uchida H, Tanaka T, Sasaki K, et al: Adenovirus-mediated transfer of siRNA against survivin induced apoptosis and attenuated tumor cell growth in vitro and in vivo. Mol Ther 10: 162-171, 2004.

12. Kikkawa K, Fujii R, Kuramoto T, et al: Dendritic cells with transduced survivin gene induce specific cytotoxic $\mathrm{T}$ lymphocytes in human urologic cancer cell lines. Urology 74 222-228, 2009

13. Schmitz M, Diestelkoetter P, Weigle B, et al: Generation of survivin-specific $\mathrm{CD}^{+} \mathrm{T}$ effector cells by dendritic cells pulsed with protein or selected peptides. Cancer Res 60: 4845-4849, 2000 .
14. Dauer M, Obermaier B, Herten J, et al: Mature dendritic cells derived from human monocytes within $48 \mathrm{~h}$ : a novel strategy for dendritic cell differentiation from blood precursors. J Immunol 170: 4069-4076, 2003.

15. Xie Z, Chen Y, Li Z, et al: Smad6 promotes neuronal differentiation in the intermediate zone of the dorsal neural tube by inhibition of the Wnt/beta-catenin pathway. Proc Natl Acad Sci USA 108: 12119-12124, 2011.

16. Dauer M, Obermaier B, Herten J, et al: Mature dendritic cells derived from human monocytes within 48 hours: a novel strategy for dendritic cell differentiation from blood precursors. J Immunol 170: 4069-4076, 2003.

17. Ziegler-Heitbrock L: The CD14+ CD16+ blood monocytes: their role in infection and inflammation. J Leukoc Biol 81: 584-592, 2007.

18. Abdi K, Singh NJ and Matzinger P: Lipopolysaccharide-activated dendritic cells: "exhausted" or alert and waiting? J Immunol 188: 5981-5989, 2012

19. Mukherjee S, Maiti PK and Nandi D: Role of CD80, CD86, and CTLA4 on mouse CD4(+) T lymphocytes in enhancing cell-cycle progression and survival after activation with PMA and ionomycin. J Leukoc Biol 72: 921-931, 2002.

20. O'Neill DW, Adams S and Bhardwaj N: Manipulating dendritic cell biology for the active immunotherapy of cancer. Blood 104: 2235-2246, 2004.

21. Zhong H, Shurin MR and Han B: Optimizing dendritic cell-based immunotherapy for cancer. Expert Rev Vaccines 6: 333-345, 2007.

22. Shurin MR: Preparation of human dendritic cells for tumor vaccination. Method Mol Biol 215: 437-462, 2003.

23. Jeras $M$, Bergant $M$ and Repnik U: In vitro preparation and functional assessment of human monocyte-derived dendritic cells-potential antigen-specific modulators of in vivo immune responses. Transpl Immunol 14: 231-244, 2005.

24. McIlroy D and Gregoire M: Optimizing dendritic cell-based anticancer immunotherapy: maturation state does have clinical impact. Cancer Immunol Immunother 52: 583-591, 2003.

25. Li F, Ambrosini G, Chu EY, et al: Control of apoptosis and mitotic spindle checkpoint by survivin. Nature 396: 580-584, 1998. 